\title{
Intraoperative blood loss during surgical treatment of low-rectal cancer by abdominosacral resection is higher than during extra-levator abdominosacral amputation of the rectum
}

Marek Bębenek

$1^{\text {st }}$ Department of Surgical Oncology, Regional Comprehensive Cancer Center, Wroclaw, Poland

Submitted: 3 May 2011

Accepted: 25 November 2011

Arch Med Sci 2014; 10, 2: 300-305

DOI: 10.5114/aoms.2014.42582

Copyright @ 2014 Termedia \& Banach

\section{Abstract}

Introduction: Abdominosacral resection (ASR) usually required blood transfusions, which are virtually no longer in use in the modified abdominosacral amputation of the rectum (ASAR). The aim of this study was to compare the intra-operative bleeding in low-rectal patients subjected to ASR or ASAR.

Material and methods: The study included low-rectal cancer patients subjected to ASR $(n=114)$ or ASAR $(n=46)$ who were retrospectively compared in terms of: 1 ) the frequency of blood transfusions during surgery and up to $24 \mathrm{~h}$ thereafter; 2 ) the volume of intraoperative blood loss ( $\mathrm{ml}$ of blood transfused) during surgery and up to $24 \mathrm{~h}$ thereafter; 3 ) hemoglobin concentrations ( $\mathrm{Hb}) 1,3$ and 5 days after surgery; 4 ) the duration of hospitalization. Results: Blood transfusions were necessary in 107 ASR patients but in none of those subjected to ASAR $(p<0.001)$. Median blood loss in the ASR group was $800 \mathrm{ml}$ (range: 100-4500 ml). The differences between the groups in median $\mathrm{Hb}$ determined 1,3 and 5 days following surgery were insignificant. The proportions of patients with abnormal values of $\mathrm{Hb}$, however, were significantly higher in the ASR group on postoperative days 1 and 3 (day 1:71.9\% vs. $19.6 \%$ in the ASAR group, $p=0.025$; day $3: 57 . \%$ vs. $13.0 \%, p=0.009$ ). Average postoperative hospitalization in ASR patients was 13 days compared to 9 days in the ASAR group ( $p=0.031)$.

Conclusions: Abdominosacral amputation of the rectum predominates over ASR in terms of the prevention of intra- and postoperative bleeding due to the properly defined surgical plane in low-rectal cancer patients.

Key words: rectal cancer, blood loss, abdominosacral amputation, total mesorectal excision.

\section{Introduction}

The large intestine is the second leading cancer location and colorectal cancer remains the second oncological reason for deaths amongst men and women from developed countries. Continuous growing trends of incidence suggest that in future decades colorectal malignancies will still constitute one of the main challenges of modern oncology. From the viewpoint of surgical oncology rectal cancers are considered a particular problem amongst the colorectal malignancies [1-3].

\author{
Corresponding author: \\ Ass. Prof. Marek Bębenek MD \\ $1^{\text {st }}$ Department \\ of Surgical Oncology \\ Regional Comprehensive \\ Cancer Center \\ pl. Hirszfelda 12 \\ 53-413 Wroclaw, Poland \\ Phone: +48713689300 \\ Fax: +48 713689309 \\ E-mail: bebmar@dco.com.pl
}


The prognosis in low-rectal cancer cases, i.e. with the tumor located up to $5 \mathrm{~cm}$ from the anorectal junction, was much less favorable as compared to mid- and upper-rectal cancer patients [4]. This resulted from the limited access to the surgical field by the routinely applied technique of abdominoperineal resection (APR) $[5,6]$. The technique usually does not allow for optimal, sharp resection, under direct vision, of the primary tumor, and consequently, markedly increases the risk of local recurrence [7].

Our previous experiences in turn indicate that abdominosacral amputation of the rectum (ASAR) is the optimal method for surgical treatment in low-rectal cancer patients. This extralevator technique, details of which were described in our previous papers, enables free access to the surgical field with the possibility of sharp and under-direct-vision complete resection of the rectum with the surrounding tissues, and allows for vascular control with immediate hemostasis. Consequently, the results achieved by us in the surgical treatment of low-rectal cancers are similar to those observed in mid- and upper-rectal cancer patients treated by means of anterior resection (AR) [8-11].

The sacral approach to the low rectum proposed by Kocher and Kraske more than a century ago $[12,13]$ was implemented in our clinic in the 1950s, initially within the technique of so-called abdominosacral resection (ASR). Work-up to the optimal methodology, however, took us several years and finally resulted in the elaborated ASAR, which combines the sacral approach with the rules of total mesorectal excision (TME) proposed by Heald et al. [14]. Efficiency of ASAR was proved by the results of our comparative studies, which showed that implementation of TME rules and the abdominosacral approach resulted in marked improvement of 5-year survival and significantly decreased the frequency of postoperative morbidity and local recurrences [15].

Comparing the archival histories of patients treated by means of non-TME ASR with the current data of subjects operated on by ASAR, we have also considered the question of intraoperative blood loss. The application of the traditional technique was usually related to the necessity of blood transfusions to low-rectal cancer patients, whereas these transfusions are virtually no longer in use in the modified ASAR surgery. We decided to verify this observation by carrying out another comparative study.

Consequently, the purpose of this study was to compare the severity of intraoperative blood loss in low-rectal patients treated by means of nonTME ASR on one hand and by ASAR technique following the TME rules on the other.

\section{Material and methods}

\section{Patients}

Low-rectal cancer patients (i.e. with lesions located less than $5 \mathrm{~cm}$ from the anorectal junction), qualified for surgery and operated on by the same surgical team at the Regional Comprehensive Cancer Center in Wroclaw between January $1^{\text {st }}, 1982$ and December 31 ${ }^{\text {st }}, 1997$ (group A, $n=$ 144) and between May $5^{\text {th }}, 1998$ and April 30 ${ }^{\text {th }}$, 2003 (group $B, n=68$ ), were subjected to analysis. The location of the rectal tumor, measured from the anal verge $(\mathrm{cm})$, as well as tumor penetration depth and local lymph node involvement, were determined in all patients.

\section{Ethics}

All the procedures were approved by the Local Ethics Committee of the Wroclaw Medical University, and the patients gave their informed consent before the start of any procedure.

\section{The old technique of non-TME ASR}

In the group A patients, following a colonic lavage and antibiotic bowel preparation (oral sulfonamide), with the patient in the supine position, a midline laparotomy was performed. After identification and division of the inferior mesenteric artery and vein, the bowel was divided at the sigmoid colon. After permanent colostomy formation, the specimen was placed in traction to simplify entering the endopelvic fascia. In order to enter the endopelvic fascia the pelvic peritoneum was incised around the rectal stump. Following ureter visualization and the division of the presacral space at the level of the sacral promontory, a combined sharp and blunt dissection was performed by inserting the hand into the loose areolar tissue between the mesorectum and sacral promontory up to the pelvic floor. The dissection was performed without direct vision and without visualization of the hypogastric nerves and autonomic nerve plexus along the pelvic wall sides surrounding the middle rectal artery. In all cases the so-called lateral ligaments were recognized, clamped, divided and ligated. When the specimen was mobilized to the floor of the pelvis - anteriorly at the retrovesical space and posteriorly at the level of the coccyx - the abdominal stage was finished. The specimen with a small swab stitched onto the end of the rectal stump was placed in the minor pelvis, with the swab positioned on the sacral side. The reperitonealization of the minor pelvis above the rectal specimen and closing of the abdominal wall finished the anterior part of the dissection. The posterior part of the dissection was carried out with the patient turned 
and placed in the prone jack-knife position. The incision around the anus was prolonged towards the sacral bone approximately to the S3-S4 level. After separating S4 or S5 with the coccyx and the dissection of Waldeyer's fascia, the rectal stump with the tumor was removed from the pelvis. The dead space under the reperitonealized pelvis floor was plugged with two long gauze pads. Finally the perineosacral wound was closed, with a drainage tube and two swabs applied to fill up the empty space and, in two layers, catgut sutures to the fat and silk to the skin were used. The drain was removed 2 to 3 days following surgery and the tampons between 7 to 12 days (Figure 1).

\section{Current ASAR technique}

In the group $B$ patients, after the initial abdominal stage of operation (TME technique) and colostomy formation, the rectal stump together with the tumor is left in the minor pelvis, and the abdominal cavity is closed in multiple layers. The patient is placed in a prone jackknife position and the anus is stitched. Subsequently, the skin is incised around the anus and the cut is elongated towards the sacral bone. The insertions of glutei

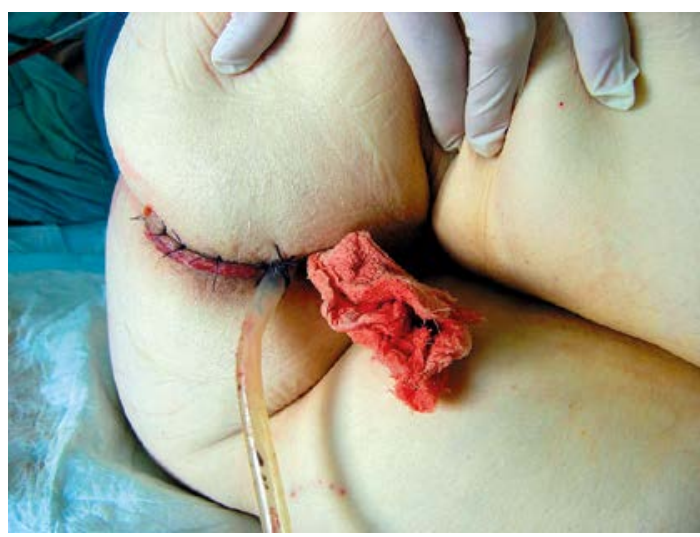

Figure 1. Surgical wound dressed with a drainage tube and tampons after non-TME ASR

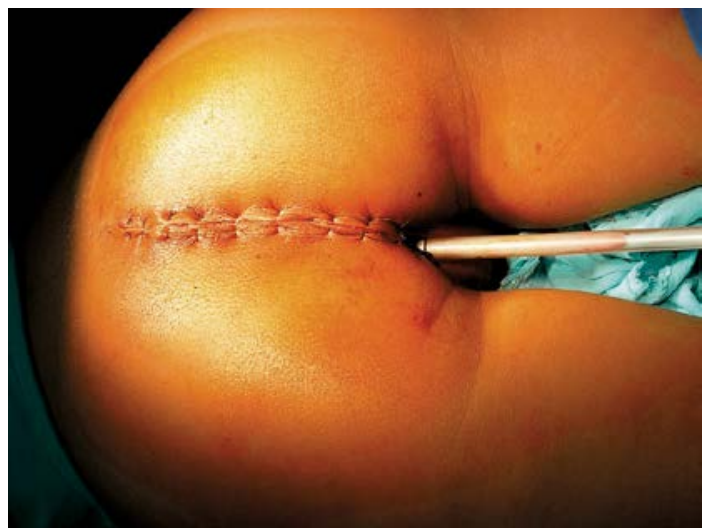

Figure 2. Surgical wound dressed with a drain after ASAR muscles are cut from the last two sacral vertebrae. The coccygeal bone or the last sacral vertebra is separated with the aid of a gouge and removed. Subsequently, following the coccygectomy or sacrectomy (S5), the presacral (Waldeyer's) fascia and parietal lamina of the pelvic fascia are cut. The rectal stump together with the tumor and surrounding mesorectum are removed via the resulting opening. Subsequently, the anal levators are cut with the simultaneous ligation of surrounding vessels. The rectum is cut off from the prostate or vagina under direct vision. In cases of adherence or invasion of the primary tumor to those organs, an en bloc resection is performed whenever feasible. The perineal wound is closed in multiple layers with a drain left in for 2-3 days (Figure 2).

\section{Result analysis}

Exclusion criteria from the analysis included: 1) stage I (Dukes A) cases, 2) suboptimal (R1 and R2) local resections [16] or metastatic disease found at surgery, 3) cases in which the hemoglobin concentrations on admission to surgical treatment were below the reference values $(7.4 \mathrm{mmol} / \mathrm{l}$ and $8.0 \mathrm{mmol} / \mathrm{l}$ in women and men respectively), 4) preoperative radiotherapy or radiochemotherapy, and 5) cases lost to follow-up or incomplete follow-up documentation.

\section{Intraoperative blood loss}

The following parameters were considered during analysis: 1) the frequency of the transfusions of blood or its preparations performed during surgery and up to $24 \mathrm{~h}$ thereafter, 2) the volume of intraoperative blood loss understood as the amount of blood transfused during surgery and up to $24 \mathrm{~h}$ thereafter, 3) hemoglobin concentrations determined 1,3 and 5 days after surgery, and 4) the duration of hospitalization. Moreover, the percentages of 5-year overall (observed and relative) survival as well as the frequencies of local recurrence were determined for all patients. Overall survival was defined as the time from the date of primary treatment to the date of death, whereas local recurrence was defined as the presence of a tumor in the pelvis or perineum.

\section{Statistical analysis}

Categorical variables were compared with the $\chi^{2}$ test, continuous variables with the Mann-Whitney $U$ test, when appropriate. Survival rates were calculated by the Kaplan-Meier method and compared with the log-rank test. Calculations were performed using Statistica 5, Version 97 (StatSoft ${ }^{\oplus}$, Poland) software, and statistical significance was defined as $p \leq 0.05$. 
Table I. Characteristics of low-rectal cancer patients subjected to non-TME ASR or ASAR

\begin{tabular}{|lccc|}
\hline Variable & Non-TME ASR $(n=114)$ & ASAR $(n=46)$ & Value of $p$ \\
\hline Age, median (range) & $65(33-84)$ & $68(39-81)$ & 0.292 \\
\hline Males, $n(\%)$ & $69(60.5)$ & $28(60.9)$ & 0.921 \\
\hline T3-T4, N1-N2 (Dukes C), $n(\%)$ & $50(43.9)$ & $21(45.7)$ & 0.473 \\
\hline T3-T4, N0 (Dukes B), $n(\%)$ & $64(56.1)$ & $25(54.3)$ & 0.471 \\
\hline
\end{tabular}

Table II. Blood parameters of low-rectal cancer patients subjected to non-TME ASR or ASAR

\begin{tabular}{|c|c|c|c|}
\hline Variable & Non-TME ASR $(n=114)$ & ASAR $(n=46)$ & Value of $p$ \\
\hline Preoperative Hb, median (range) & $11.2(10.0-15.4)$ & $11.4(9.9-15.0)$ & 0.543 \\
\hline Intraoperative transfusion, $n(\%)$ & $107(93.9)$ & $0(0.0)$ & $<0.001$ \\
\hline Blood loss, median (range) $[\mathrm{ml}]$ & $800(100-4500)$ & $250(50-900)^{*}$ & 0.012 \\
\hline $\mathrm{Hb}-1$ day after surgery, median (range) & $9.6(6.1-12.4)$ & $11.2(7.0-13.7)$ & 0.063 \\
\hline $\mathrm{Hb}-3$ days after surgery, median (range) & $10.1(7.0-14.0)$ & $12.1(7.2-14.2)$ & 0.069 \\
\hline $\mathrm{Hb}-5$ days after surgery, median (range) & $10.8(7.1-14.5)$ & $11.3(7.3-14.9)$ & 0.088 \\
\hline Abnormal ${ }^{* *} \mathrm{Hb}-1$ day after surgery, $n(\%)$ & $82(71.9)$ & $9(19.6)$ & 0.025 \\
\hline Abnormal ${ }^{* *} \mathrm{Hb}-3$ days after surgery, $n(\%)$ & $65(57.0)$ & $6(13.0)$ & 0.009 \\
\hline Abnormal $^{* \star} \mathrm{Hb}-5$ days after surgery, $n(\%)$ & $3(2.6)$ & $1(2.2)$ & 0.982 \\
\hline
\end{tabular}

*Volume as assessed by a surgeon intraoperatively, ${ }^{\star \star}$ hemoglobin concentration below $7.4 \mathrm{mmol} / \mathrm{l}$ and $8.0 \mathrm{mmol} / \mathrm{l}$ in women and men respectively

\section{Results}

One-hundred and sixty patients, including 114 cases operated on by means of ASR and 46 subjected to ASAR surgery, were qualified for further analysis based on the exclusion criteria defined. Both groups were comparable in terms of distributions of age, gender, clinical stages, and the average values of preoperative hemoglobin concentrations (Tables I and II).

Intraoperative blood transfusions were necessary in almost all patients operated on by ASR, whereas there was no need for them in any case subjected to ASAR. Median blood loss in the ASR group was $800 \mathrm{ml}$ (Table II), with individual values ranging from $100 \mathrm{ml}$ to $4500 \mathrm{ml}$ (Figure 3).

The differences between ASR patients and those operated on by means of ASAR in the median hemoglobin concentrations determined 1, 3 and 5 days following surgery were found to be insignificant (Table II).

On postoperative days 1 and 3, the proportions of ASR patients with abnormal hemoglobin concentration, i.e. below $7.4 \mathrm{mmol} / \mathrm{l}$ and $8.0 \mathrm{mmol} / \mathrm{l}$ in women and men respectively, were significantly higher compared to those operated on by means of ASAR. The groups were not significantly different, however, when compared on day 5 (Table II).

Therapeutic results achieved in patients of both groups are summarized in Table III. Three cases of perioperative mortality occurred in ASR patients - all noted during the initial $48 \mathrm{~h}$ following surgery. All of them resulted from massive blood loss. The latter group was also significantly worse in terms of perioperative morbidity $(40.4 \%$ vs. $10.9 \%$ in ASAR patients). Average time of postoperative hospitalization in patients operated on by means of ASR was 13 days and was markedly

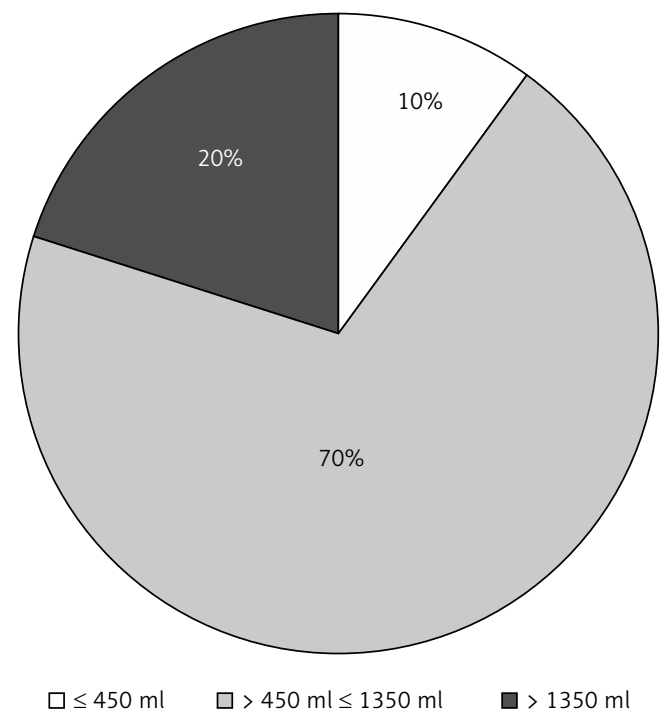

Figure 3. Distribution of non-TME ASR patients who required intraoperative transfusions of blood 
Table III. Therapeutic results of low-rectal cancer patients subjected to non-TME ASR or ASAR

\begin{tabular}{|lccc|}
\hline Variable & Non-TME ASR & ASAR & Value of $p$ \\
\hline Perioperative mortality, $n(\%)$ & $3(2.6)$ & 0 & $<0.001$ \\
\hline Morbidity, $n(\%)$ & $46(40.4)$ & $5(10.9)$ & $<0.001$ \\
\hline Postsurgical hospitalization, median (range) [days] & $13(9-21)$ & $9(6-15)$ & 0.031 \\
\hline 5-year observed survival, $n(\%)$ & $44(38.6)$ & $31(67.4)$ & 0.024 \\
\hline 5-year relative survival (\%) & 44.5 & 72.7 & 0.017 \\
\hline Local recurrence rate, $n(\%)$ & $32(28.1)$ & $2(4.3)$ & 0.003 \\
\hline
\end{tabular}

longer than in cases in which the ASAR technique was implemented (9 days on average). Finally, ASAR patients outnumbered those operated on by means of ASR in terms of 5-year observed (67.4\% vs. $38.6 \%)$ and relative survival (72.7\% vs. $44.5 \%)$ and had a significantly lower local recurrence rate (4.3\% vs. $28.1 \%)$.

\section{Discussion}

As previously mentioned, the sacral approach in the prone jackknife position facilitates maximal exposure for complete resection and enables unlimited surgical access, under direct vision, to the pelvic cavity along with vascular control. This results in both the optimal resection of the malignancy and the proper identification of blood vessels and immediate hemostasis. Nevertheless, heavy bleeding from the rectum-surrounding tissues, especially from the presacral veins localized under the sacral fascia or injured mesorectum, did occur during the abdominal stage of non-TME ASR surgery. The positive implications of ASAR are related to the fact that TME components were included in the surgical protocol. Consequently the surgical specimen of the rectum that is removed is not torn, and it is possible to avoid excessive bleeding during the abdominal stage of the operation.

Studies on the role played by TME implementation in the reduction of perioperative blood loss are scant and their results are quite unequivocal. There are some large studies, however, whose results indicate that the perioperative blood loss was reduced by up to $600-800 \mathrm{ml}$ due to the implementation of TME procedures [17-19]. A similar association refers to the frequency of perioperative blood transfusions performed in rectal cancer patients operated on with the aid of the TME-including procedure $[17,19,20]$. Hence, all the aforementioned results are consonant with the ones revealed by our present analysis.

Both our present results and those of our previous comparative study indicate that the therapeutic results achieved by ASAR are markedly better compared to previous variants of ASR [15]. The present study, however, underlines another advantage of ASAR, namely the markedly reduced intraoperative bleeding and - which is more important - no need for blood transfusions. Many studies have revealed that blood transfusions negatively affect the prognosis in rectal cancer patients by increasing the rate of local recurrences and reducing long-term survival. This phenomenon plausibly results from the adverse effects of transfusion-induced suppression of the immune system with its consequent lack of resistance to tumor growth [21]. A higher rate of local recurrence and reduction in long-term survival following surgery in rectal cancer patients who were given perioperative blood transfusions has been proved by several studies [22-26]. A causal relationship between an intraoperative blood transfusion and the risk of local recurrence was also revealed through meta-analyses by Amato and Pescatori $[27,28]$. Although there exist some studies whose results suggest that blood transfusions per se do not worsen prognosis in rectal cancer patients [29-32], their frequency should be reduced - at least because of the established effect of transfusion-derived immunosuppression on the increased susceptibility of patients to bacterial infections [33, 34].

Exclusion of patients with suboptimal resections is one potential limitation of this study since one can expect more severe bleeding in such cases. Unfortunately, proportions of suboptimal resections were too small and unequally distributed between the ASR and ASAR group.

In conclusion, the present study revealed that the blood loss resulting from modern ASAR surgery is so small that it does not require intraoperative transfusions of blood or its preparation. This was also proved by significantly higher hemoglobin concentrations found in patients who were operated on by ASAR technique and by the shorter postoperative hospital stays they required compared to the ASR group.

The present study revealed another positive aspect of ASAR in the surgical treatment of low-rectal cancer cases, namely the prevention of intra- and postoperative bleeding as the result of a properly defined surgical plane. 


\section{References}

1. Smolińska K, Paluszkiewicz P. Risk of colorectal cancer in relation to frequency and total amount of red meat consumption. Systematic review and meta-analysis. Arch Med Sci 2010; 6: 605-10.

2. Stec R, Pławski A, Synowiec A, Mączewski M, Szczylik C. Colorectal cancer in the course of familial adenomatous polyposis syndrome ("de novo" pathogenic mutation of APC gene): case report, review of the literature and genetic commentary. Arch Med Sci 2010; 6: 283-7.

3. Klimczak A, Kempińska-Mirosławska B, Mik M, Dziki Ł. Dziki A. Incidence of colorectal cancer in Poland in 1999-2008. Arch Med Sci 2011; 7: 673-8.

4. Nagtegaal ID, van de Velde CJH, Marijnen CAM, van Krieken JHJM, Quirke P. Low rectal cancer: a call for a change of approach in abdominoperineal resection. J Clin Oncol 2005; 23: 9257-64.

5. Miles WE. A method of performing abdominoperineal excision for carcinoma of the rectum of the terminal portion of the pelvic colon. Lancet 1908; 2: 1812-3.

6. Lloyd-Davies OV, Lond MS. Lithotomy-Trendelenburg position for resection of rectum and lower pelvic colon. Lancet 1939; 234: 74-6.

7. Quirke P, Durdey P, Dixon MF, Williams NS. Local recurrence of rectal adenocarcinoma due to inadequate surgical resection: histopathological study of lateral tumor spread and surgical excision. Lancet 1986; 2: 996-9.

8. Bębenek M, Pudełko M, Cisarż K, et al. Therapeutic results in low-rectal cancer patients treated with abdominosacral resection are similar to those obtained by means of anterior resection in mid- and upper-rectal cancer cases. Eur J Surg Oncol 2007; 33: 320-3.

9. Bębenek M. Abdominosacral resection (ASR) is not related to the risk of neurological complications in low-rectal cancer patients. Colorectal Dis 2009; 11: 373-6.

10. Bębenek M. Abdominosacral amputation of the rectum for low-rectal cancer: ten years of experience. Ann Surg Oncol 2009; 16: 2211-7.

11. Bębenek M. Influence of neoadjuvant radio(chemo) therapy on wound healing in lower-rectal cancer patients subjected to abdominosacral resection (ASR). World J Colorectal Surg 2008; 1 [http://services.bepress. com/wjcs/vol1/iss1/art10].

12. Liebermann-Meffert D. History of the International Society of Surgery/Societe International de Chirurgie (ISS/ SIC). I. Short story of Theodor Kocher's life and relationship to the International Society of Surgery. World J Surg 2000; 24: 2-9.

13. Classic articles in colonic and rectal surgery. Paul Kraske 1851-1930. Extirpation of high carcinomas of the large bowel. Dis Colon Rectum 1984; 27: 499-503.

14. Heald RJ, Husband EM, Ryall RD. The mesorectum in rectal cancer surgery - the clue to pelvic recurrence? $\mathrm{Br}$ J Surg 1982; 69: 613-6.

15. Bębenek M, Rząca M. Total mesorectal excision (TME) enhances the effectiveness of the abdominosacral resection of the rectum (ASAR) in low-rectal cancer patients. Nowotwory J Oncol 2009; 59: 16-21.

16. Compton CC, Fielding LP, Burgart LJ, et al. Prognostic factors in colorectal cancer. College of American Pathologists Consensus Statement 1999. Arch Pathol Lab Med 2000; 124: 979-94.

17. Smedh K, Olsson L, Johansson H, Aberg C, Andersson M. Reduction of postoperative morbidity and mortality in patients with rectal cancer following the introduction of a colorectal unit. Br J Surg 2001; 88: 273-7.
18. Nesbakken A, Nygaard K, Westerheim O, Lunde OC, Mala T. Audit of intraoperative and early postoperative complications after introduction of mesorectal excision for rectal cancer. Eur J Surg 2002; 168: 229-35.

19. Mynster T, Nielsen HJ, Harling H, Bülow S; Danish TMEgroup, RANX05-group. Blood loss and transfusion after total mesorectal excision and conventional rectal cancer surgery. Colorectal Dis 2004; 6: 452-7.

20. Dowdall JF, Maguire D, McAnena OJ. Experience of surgery for rectal cancer with total mesorectal excision in a general surgical practice. Br J Surg 2002; 89: 1014-9.

21. Blajchman MA, Bordin JO. The tumor growth-promoting effect of allogeneic blood transfusions. Immunol Invest 1995; 24: 311-7.

22. Tartter PI. The association of perioperative blood transfusion with colorectal cancer recurrence. Ann Surg 1992; 216: 633-8.

23. Houbiers JG, Brand A, van de Watering LM, et al. Randomised controlled trial comparing transfusion of leucocyte-depleted or buffy-coat-depleted blood in surgery for colorectal cancer. Lancet 1994; 344: 573-8.

24. Edna TH, Bjerkeset T. Perioperative blood transfusions reduce long-term survival following surgery for colorectal cancer. Dis Colon Rectum 1998; 41: 451-9.

25. Nakafusa Y, Tanaka T, Tanaka M, Kitajima Y, Sato S, Miyazaki K. Comparison of multivisceral resection and standard operation for locally advanced colorectal cancer: analysis of prognostic factors for short-term and longterm outcome. Dis Colon Rectum 2004; 47: 2055-63.

26. Miki C, Hiro J, Ojima E, Inoue Y, Mohri Y, Kusunoki M. Perioperative allogeneic blood transfusion, the related cytokine response and long-term survival after potentially curative resection of colorectal cancer. Clin Oncol 2006; 18: 60-6.

27. Amato AC, Pescatori M. Effect of perioperative blood transfusions on recurrence of colorectal cancer: meta-analysis stratified on risk factors. Dis Colon Rectum 1998; 41: 570-85.

28. Amato A, Pescatori M. Perioperative blood transfusions for the recurrence of colorectal cancer. Cochrane Database Syst Rev 2006; 1: CD005033.

29. Donohue JH, Williams S, Cha S, et al. Perioperative blood transfusions do not affect disease recurrence of patients undergoing curative resection of colorectal carcinoma: a Mayo/North Central Cancer Treatment Group study. J Clin Oncol 1995; 13: 1671-8.

30. Marquet RL, Busch OR, Jeekel J, Heiss MM, Amato AC. Are allogeneic blood transfusions acceptable in elective surgery in colorectal carcinoma? Eur J Cancer 1999; 35: 352-60.

31. Mynster T, Christensen IJ, Moesgaard F, Nielsen HJ. Effects of the combination of blood transfusion and postoperative infectious complications on prognosis after surgery for colorectal cancer. Danish RANX05 Colorectal Cancer Study Group. Br J Surg 2000; 87: 1553-62.

32. Dionigi G, Rovera F, Boni L, et al. The impact of perioperative blood transfusion on clinical outcomes in colorectal surgery. Surg Oncol 2007; 16 (Suppl. 1): S177-82.

33. Nielsen HJ. Detrimental effects of perioperative blood transfusion. Br J Surg 1995; 82: 582-7.

34. Jensen LS, Hokland M, Nielsen HJ. A randomized controlled study of the effect of bedside leucocyte depletion on the immunosuppressive effect of whole blood transfusion in patients undergoing elective colorectal surgery. Br J Surg 1996; 83: 973-7. 